\title{
Application of Estimation Techniques on Queue Lengths Estimation in Traffic Network
}

\author{
Pavla Pecherková \\ Research Centre Data - Algorithms - Decision Making \\ Department of Adaptive Systems, Institute of Information \\ Theory and Automation of the Academy of Sciences of the Czech \\ Republic, Pod vodárenskou věží 4, 182 08, Prague, Czech Republic \\ Miroslav Flídr and Jindřich Duník \\ Research Centre Data - Algorithms - Decision Making \\ Department of Cybernetics, Faculty of Applied Sciences, \\ University of West Bohemia, \\ Univerzitní 8, 30614 Plzeň, Czech Republic
}

\begin{abstract}
The paper deals with the application of the state and parameter estimation techniques in the area of traffic control. The most important properties of the traffic system are described and the model of the traffic system, based on the traffic flow conservation principle, is presented. Various estimation and identification techniques are briefly introduced and applied for three types of roads and micro-regions, namely for city ring road, peripheral road, and city centre. Performance of estimation techniques is validated, using the derived models on real and synthetic data coming from Prague, with respect to accuracy and complexity.
\end{abstract}

Index Terms-traffic system model, stochastic systems, state estimation, Kalman filtering

\section{INTRODUCTION}

Number of vehicles on streets is increasing every day. In order to preserve or to improve throughput of current light controlled network it is necessary to employ an intelligent dynamic traffic control.

The dynamic traffic control, contrary to the static control, depends not only on the time plan but also on the actual traffic flow. The knowledge of actual traffic flow allows controlling traffic system via suitable settings of parameters of signal traffic lights. Often used representatives of the dynamic traffic control are the traffic responsive control with optimisation and the adaptive control. The traffic responsive control with optimisation, e.g. systems SCOOT and SCATS, are characteristic by presence of one traffic centre performing optimisation of the traffic light parameters of a whole area in short time period [1], [2], [3]. This induces a main disadvantage of such systems which lies in the demands on the communication network throughput. To rectify this problem the adaptive systems, such as MOTION, have been proposed [4], which allow to locally control smaller areas (e.g. particular intersections) in short time period and to optimise a whole area in the significantly longer time periods. In Prague, Czech Republic, the dynamic traffic control MOTION was tested [5]. Unfortunately, the tests showed that MOTION is sensitive to habits of local drivers. These habits differ from country to country. In case of Prague, the system MOTION sets to long cycle times of traffic lights. For this reason the novel non-commercial adaptive traffic control system has been proposed [6], [7].

This work was supported by the Ministry of Education, Youth and Sports of the Czech Republic, project No. 1M0572.
This novel adaptive traffic control system is being developed especially for the needs of cities with old urban areas, which are characterised by narrow and short roads, one-way roads, and by large number of both uncontrolled and controlled intersections. However, it is also possible to apply it to all other traffic areas, such as city ring roads and peripheral roads. The control system is based on the minimisation of the total length of vehicle queues on all roads.

The knowledge of queue lengths represents the best information about the real situation in the traffic network. However, nowadays, when many intersection arms are equipped with traffic detectors ${ }^{1}$, the lengths of vehicle queues are not directly measurable and it is necessary to estimate them on the basis of measurable traffic quantities such as intensity, occupancy, density, etc.

The knowledge of the queue lengths is essential for efficient control. Thus, it is necessary to supplement the control system with queue length estimation subsystem. The cornerstone of the queue length estimation is a sufficiently exact model of an intersection or a micro-region ${ }^{2}$. Therefore, the technique for design of the traffic state space model, where the directly immeasurable queue lengths are included in the state, has been developed [6], [7]. The traffic system can be sufficiently described by a linear model and so the well-known Kalman filter [8] can be used as a state estimator. The parameters of the model are usually determined off-line on the basis of physical properties of the traffic system, on expert knowledge and/or analysis of measured data. The off-line identification of parameters is suitable in the cases when the traffic intensity is almost constant during long interval, for example, on a ring

\footnotetext{
${ }^{1}$ The traffic detectors are used for collecting statistics about traffic flow. The road (or lane) can be equipped with one detector on the output and three detectors on the input: (i) detector on the stop line, (ii) outlying detector (30 $\mathrm{m}$ before the stop line), (iii) strategic detector - (100 $\mathrm{m}$ before the stop line) Ideally, each lane has all three types of the detectors but in real traffic system, the lane is usually equipped with one or two types of such detector, due to the constrained conditions e.g. in the old urban areas with short lanes. The strategic detectors, which are the most remote from a stop line, give the best information about the traffic flow at present. Unfortunately, the detector cannot provide any useful information about the real traffic flow if the queue length is greater then the distance between stop line and the detector placement.

${ }^{2} \mathrm{~A}$ micro-region consists of several intersections with some detectors on the input and output roads. There must be at least one signal-controlled intersection.
} 
road. In other cases, the on-line identification of parameters is needed to obtain the accurate parameter values corresponding to actual traffic situation at each time instant. Such simultaneous estimation of the state and parameters leads to the application of the nonlinear techniques either local, such as the extended Kalman filter or the derivative-free Kalman filters [8], [9], [10], or global, e.g. the Gaussian sum filter [11], [12].

The goal of the paper is to test different estimation methods for estimation of immeasurable queue lengths and unknown parameters. These tests will be performed on various types of the traffic systems with respect to the needs of the traffic control (accuracy and computational demands). The estimation methods are validated using the derived models on real and synthetic data.

The paper is organised as follows. Section 2 gives brief description of traffic model design technique. In Section 3 various estimation methods are shortly discussed. Then, estimation methods are applied on the several different types of roads or micro-regions in Section 4. Finally, Section 5 includes some concluding remarks.

\section{TRAFFIC MODEL}

This section will be devoted to the description of the traffic system model. Firstly, the basic quantities that describe the traffic system will be introduced. Secondly, the model of the traffic system based on the conservation principle will be presented.

The quantities characterising the traffic system can be divided into two basic groups:

(i) Quantities determined by the intersection layout and configuration

- Saturated flow $\mathcal{S}$ is the maximal number of vehicles flowing through the intersection lanes per hour - given in $[u v / h]$, where $u v$ represents unit vehicle. This quantity mainly depends on the road width, number of traffic lanes in one direction, and the turning movement.

- Turning movement $\alpha_{i, j}$ is the ratio of vehicles going from the $i$-th arm to the $j$-th arm [\%].

- Cycle time is a period of a phase change of the traffic light $[s]$.

- Green time ratio $z$ is the ratio of the effective green time of a phase to the cycle time [\%].

- Offset is the difference between the start (or end) of green times at the two adjacent signalized intersection $[s]$.

(ii) Quantities describing the traffic flow

- Input intensity $\mathcal{I}_{t}$ or output intensity $\mathcal{Y}_{t}$ (at time instant $t$ ) is the amount of passing unit vehicles per hour $[u v / h]$ measured by the detector placed in the input or output lane, respectively.

- Occupancy $O_{t}$ is the proportion of the period when the detector is occupied by vehicles [\%].

- Queue length $\xi_{t}$ is a number of all vehicles waiting in one lane $[u v]$.

The fundamental idea of the traffic model is based on a principle of traffic flow conservation principle. For the sake of

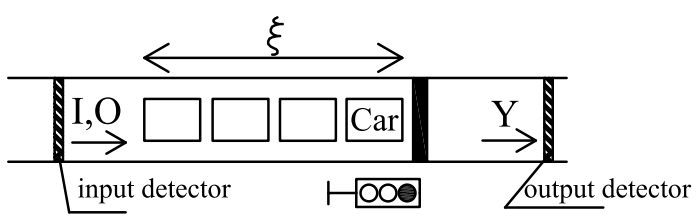

Fig. 1. An example of controlled one-way road.

simplicity, the basic design technique of the state space traffic model will be shown only on the very simplest micro-region including a road with one input and one output detector and with one traffic light as is depicted in Fig. 1.

The state of the traffic system $\mathbf{x}_{t} \in \mathbb{R}^{n_{x}}$, which completely describes the traffic situation at each time instant, is defined by the following vector

$$
\mathbf{x}_{t}=\left[x_{1, t}, x_{2, t}, x_{3, t}, x_{4, t}\right]^{T}=\left[\xi_{t}, I_{t}, Y_{t}, O_{t}\right]^{T}
$$

and the evolution of the state is described by the following dynamic equations

$$
\begin{aligned}
& x_{1, t+1}=\underbrace{\delta_{t} x_{1, t}}_{\text {previous queue }}-\underbrace{\left[\delta_{t} S+\left(1-\delta_{t}\right) x_{2, t}\right] z_{t}}_{\text {departed vehicles }}+\underbrace{x_{2, t}}_{\text {arrived vehicles }}+w_{1, t}, \\
& x_{2, t+1}=x_{2, t}+w_{2, t}, \\
& x_{3, t+1}=\left(1-\delta_{t}\right) x_{1, t}-\left[\delta_{t} S+\left(1-\delta_{t}\right) x_{2, t}\right] z_{t}+w_{3, t}, \\
& x_{4, t+1}=\kappa_{t} x_{1, t}+\beta_{t} x_{4, t}+\lambda_{t}+w_{4, t},
\end{aligned}
$$

where the quantities $I_{t}, Y_{t}$ and $S$ denote the intensities $\mathcal{I}_{t}, \mathcal{Y}_{t}$ and saturation flow $\mathcal{S}$ with respect to the measurement period, i.e. they are given in $[u v]$.

The vector of measurements $\mathbf{y}_{t} \in \mathbb{R}^{n_{y}}$, provided by the traffic detectors, is defined as

$$
\mathbf{y}_{t}=\left[x_{2, t}, x_{3, t}, x_{4, t}\right]^{T}+\mathbf{v}_{t}=\left[I_{t}, Y_{t}, O_{t}\right]^{T}+\mathbf{v}_{t} .
$$

The vectors $\mathbf{w}_{k}=\left[w_{1, k}, w_{2, k}, w_{3, k}, w_{4, k}\right]$ and $\mathbf{v}_{k}$ of appropriate dimensions represent the state and measurement noises. The noises are mutually independent with zero-mean probability density functions (pdfs).

The symbol $\delta_{t}$ denotes function that indicates whether there is a congestion on the arm which is defined as follows

$$
\delta_{t}=\left\{\begin{array}{lll}
0 & \xi_{t}+I_{t} \leq S \quad \text { (no congestion) } \\
1 & \xi_{t}+I_{t}>S \quad \text { (congestion) }
\end{array}\right.
$$

The parameters $\beta_{t}$ and $\kappa_{t}$ reflect the inherent relations between state variables and parameter $\lambda_{t}$ is a correction term to omit a zero occupancy, because the zero queue length and occupancy at the previous time instant do not imply the zero occupancy at the actual time instant. The extension of the one-lane model design to the description of an arbitrary micro-region is straightforward. Such micro-region model consists of the models of particular arms together with relations between them (e.g. turning movement). The traffic model for a general microregion can be written in the matrix form

$$
\begin{aligned}
\mathbf{x}_{t+1} & =\mathbf{A}_{t} \mathbf{x}_{t}+\mathbf{B}_{t} \mathbf{z}_{t}+\mathbf{F}_{t}+\mathbf{w}_{t}, \\
\mathbf{y}_{t} & =\mathbf{C} \mathbf{x}_{t}+\mathbf{G}_{t}+\mathbf{v}_{t},
\end{aligned}
$$


where matrices $\mathbf{A}_{t}, \mathbf{B}_{t}, \mathbf{F}_{t}, \mathbf{C}$, and $\mathbf{G}_{t}$ have appropriate dimensions, $\mathbf{z}_{t}$ is the vector of the green time ratios, and $\mathbf{w}_{k}$ and $\mathbf{v}_{k}$ are the state and measurement noises. An example of construction of traffic model of a real micro-region can be found e.g. in [7].

\section{Considered Estimation Techniques}

Application of the state estimation techniques is conditioned by the knowledge of the sufficiently exact model. However, the model presented in previous section contains several unknown parameters, in both "deterministic" and "stochastic" part, which cannot be determined from the physical properties of the traffic system, namely parameters $\beta_{t}, \kappa_{t}, \lambda_{t}$ and the statistics of the state and measurement noises. Therefore, the unknown parameters have to be identified somehow.

Generally, there are two possibilities how to estimate the state and the parameters in the deterministic part of the system. The first possibility is based on an off-line identification of the unknown parameters, e.g. by prediction error methods [13], and subsequently on an on-line estimation of the state by the Kalman Filter (KF) [8]. However, off-line identified time variant or invariant parameters represent average values rather than the actual (true) parameters and this approach is therefore suitable for traffic systems where intensity of the traffic flow is almost constant. The second possibility is based on the simultaneous on-line estimation of the state and the parameters by extension of the state with vector of the unknown parameters [14]. This leads to the nonlinear model of the traffic system and to the application of the suitable nonlinear estimation method.

There are two main groups of nonlinear estimation methods, namely local and global methods. The local methods are often based on approximation of the nonlinear functions in the state or measurement equation so that the technique of the Kalman Filter design can be used for the BRRs solution. This approach causes that all conditional pdfs of the state estimate are given by the first two moments. This rough approximation of posterior estimates induces local validity of the state estimates and consequently impossibility to ensure the convergence of the local filter estimates. The resulting estimates of the local filters are suitable mainly for point estimates. On the other hand, the advantage of the local methods can be found in the simplicity of the BRRs solution. Generally, there are two main approaches in the local filter design. The first possibility is to approximate the nonlinear function in the model by means of the Taylor expansion first or second order, which leads e.g. to the Extended Kalman Filter, or by means of the Stirling's polynomial interpolation, which leads to the Divided Difference Filter first or second order (DD1, DD2) [10], [12]. The second possibility, often used in the local filter design, is based on the approximation of state estimates by a set of deterministically chosen points. This method is known as the Unscented Transformation and its application in the local filter design leads to e.g. the Unscented Kalman Filter (UKF) [15], [12]. The global methods are rather based on approximation of the conditional pdf of the state estimate of some kind to accomplish better state estimates.

Although, the global methods are more sophisticated than local methods, they have significantly higher computation demands. Due to the computational efficiency, the stress will be mainly laid on the local methods, namely on the local derivative-free local filters methods represented by the unscented Kalman filter and divided difference filters [9], [10].

As far as the statistics of the state and measurement noises are concerned, both noises are currently supposed to be zero mean with unknown covariance matrices. The noise covariance matrices can be generally estimated online or offline. Due to the extensive computational demands of online noise covariance matrices estimation methods [16], [17], they were estimated off-line by the estimation technique based on the multi-step prediction [18] for both linear models and extended nonlinear models for various intensities of the traffic flow.

\section{COMPARISON OF Estimation TECHNiQues}

In this section, various state and parameter estimation techniques are tested and compared on three different types of traffic systems in three different situations. First, a historical city centre is considered where some of the roads are not equipped with the detectors. Second, an example with peripheral road, which is fully equipped with the detectors is treated for situation when one of detectors broke down and a possible solution of the problem is presented. Third, a problem of detection of an accident on the city ring road and its impact on the traffic flow is discussed.

\section{A. City Centre}

City centre roads are characterised by a large number of intersections, either controlled or uncontrolled, in a small area. The traffic intensity is relatively low, but due to small capacity of city centre traffic infrastructure, roads are oversaturated mainly in workweek during morning and evening peak hours. In this kind of micro-region, the main limitation is the short length of roads between two adjacent intersections. It is no exception to the rule that the queue on one intersection reaches to an adjacent intersection.

\section{Description of the Micro-Region}

The estimation of queue lengths was tested on the microregion in Prague city district Smíchov. This micro-region is composed of two four-arm controlled intersections (I1, I2) and three uncontrolled ones. The arms are one-way and they consist of several lanes, see Fig. 2. The input arms Zborovská and Matoušova are equipped with the strategic detectors. The input arm $V$ Botanice is equipped with the outlying detector. Output detectors are in the arms $V$ Botanice and Zborovská. The unmeasured lanes are connected with thoroughfare Zborovská between the first strategic detector (clockwise) and the output detector. The data are measured at 90 second intervals.

The traffic system can be described by previously introduced linear state space model given by relations (6), (7) where parameters $\kappa_{t}, \beta_{t}$, and $\lambda_{t}$ are unknown for all arms of the microregion. These parameters strongly depend on the varying traffic 


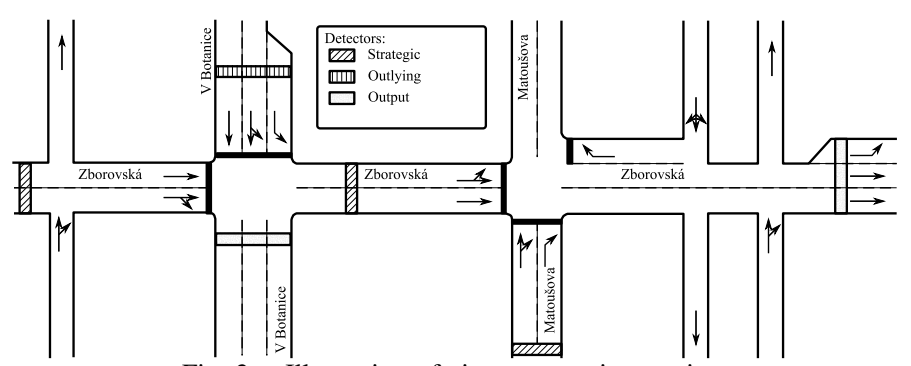

Fig. 2. Illustration of city centre micro-region.

TABLE I

COMPARISON OF DIFFERENT ESTIMATION TECHNIQUES USED FOR ESTIMATION OF QUEUE LENGTH IN THE CITY CENTRE ROAD.

\begin{tabular}{|l|c|c|c|c|}
\hline & DD1 & DD2 & UKF & KF \\
\hline \hline MSE $\left[u v^{2} \cdot 10^{4}\right]$ & 1.40 & 1.38 & 1.42 & 329.25 \\
\hline RMSE $[u v]$ & 3.88 & 3.72 & 3.75 & 32.48 \\
\hline Max. difference $[u v]$ & 14.7 & 13.9 & 15.0 & 122.1 \\
\hline Time $[s]$ & 0.249 & 0.252 & 0.260 & 0.068 \\
\hline
\end{tabular}

flow during a whole day and they should be therefore estimated on-line as well as the state of the system. On-line estimation of the state and parameters can be based on the extension of the state by the vector of unknown parameters. This extension makes the model nonlinear from the estimation point of view and thus the nonlinear estimation techniques have to be employed. In this case, the original state has dimension $\operatorname{dim}\left(\mathbf{x}_{t}\right)=36$ but the extended state, consisted of queues, occupancies, and parameters, has dimension $\operatorname{dim}\left(\tilde{\mathbf{x}}_{t}\right)=68$. The initial set of the parameters was obtained using off-line analysis of already measured data.

For the state and parameter estimation the real data, namely input and output intensities, occupancies, and cycle and green times, were used. The "true" queue lengths were determined by simulation software AIMSUN $^{3}$ and they were used for the comparison with estimated lengths. The validation and calibration process of the simulator was made with respect to the particularities of the local traffic system. The validation of the queue length reconstruction was made on several types of micro-regions in Prague in accord to the guidelines specified in [19].

\section{Comparison of the Tested Estimation Techniques}

The Kalman filter and three local filters, namely the divided difference filter first and second order and the unscented Kalman filter, were used for estimation of the queue lengths on all arms of micro-region depicted in Fig. 2. Utilisation of the $\mathrm{KF}$ is conditioned by the off-line identification of unknown parameters occurred in the deterministic part of the model, i.e. in the system matrices. These parameters were identified off-line by means of the prediction error method on the basis of previously collected data without regard to the part of a day and thus, they represent rather average values of the parameters. The simultaneous on-line estimation

${ }^{3}$ AIMSUN is a simulation software tool which is able to reproduce the traffic condition of any traffic network. It is mainly used for testing new traffic control system and management strategies, but it can be also used for traffic state prediction and other real time applications.

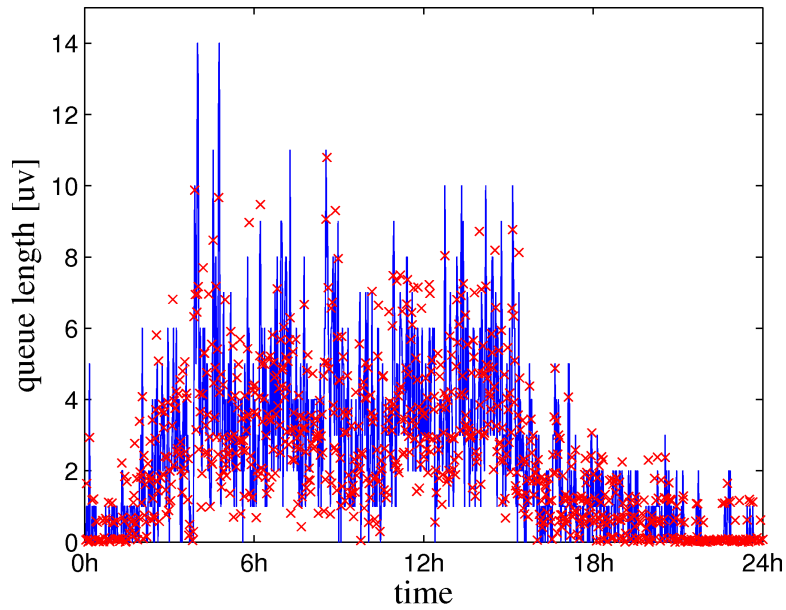

Fig. 3. True (-) and estimated (x) queue during a whole day.

of the state and parameters allows to find the traffic model representing actual traffic situation and it inevitably leads to application of nonlinear filters, represented by the UKF, DD1, and DD2. Table I shows comparison of the four filters with respect to the estimation quality and to the computation load. The estimation quality is compared on the basis of the mean square error (MSE), representing average sum squared differences between the "true" and estimated queue lengths on one arm and one day, the root mean square error (RMSE), representing an average error of estimate in one sample period, and the maximal difference, which gives information about the maximal error of estimated queue lengths. The last item in the table represents an average computational time required for estimation of queue lengths by all filters for one measurement sample. It can be seen that the KF gives noticeably worse estimation results. This is caused by the off-line identified parameters, which do not respect an actual traffic situation, and by high number of unmeasured roads.

Whereas by utilisation of the nonlinear techniques it is possible to estimate the actual values of the immeasurable intensities by appending them to the "already extended" state as well, the KF uses the typical daily courses found off-line. Unfortunately, the unmeasured roads are often characterised by the sudden and unpredictable changes of intensities, and thus the typical daily courses can be far from the actual ones. In case, that micro-region is fully covered by detectors, the significantly faster KF provides almost the same estimates as the local filters. As far as quality of local filters is consid- 


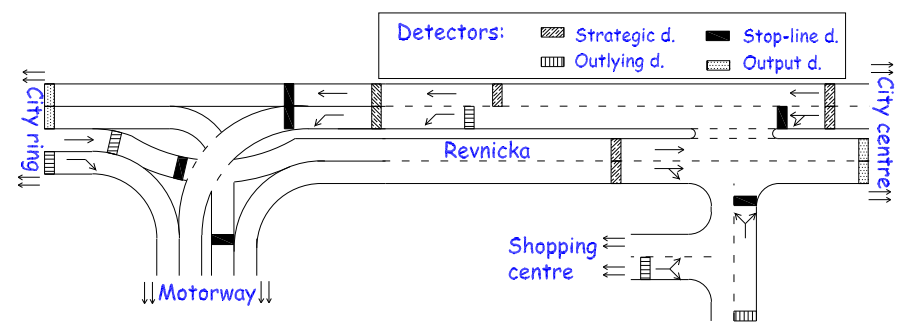

Fig. 4. Illustration of chosen Prague peripheral road.

ered, they show very similar estimation performance in the traffic problem. The reason can be found in a absence from significant nonlinearities [12]. For completeness an example of "true" and estimated course of the queue length in arm Matoušova is shown in Fig. 3. The estimates were retrieved using the DD1 method.

\section{Remarks}

The first remark concerns the estimation quality of the KF, which can be improved by the identification of unknown parameters with respect to the part of the day, i.e. for each characteristic part of the day (peak hours, peak-off hours, weekend, etc.) specific set of parameters is provided.

The second remark deals with the choice of the local estimation methods. Only the derivative-free versions were selected because they provide at least comparable results to the well known and established extended Kalman filter without the necessity to derive the derivatives of the nonlinear functions in the extended model. Such derivation would be quite tedious because the extended model has, even in this rather simpler example, dimension of the state sixty-eight.

The last remark concerns the rare situation when the queue lengths estimates have negative values. In such cases, the negative estimates are replaced by the zero value.

\section{B. Peripheral Road}

Peripheral roads are roads with high intensity of the traffic flow, but contrary to the city ring road, they run through the city centre. These roads are oversaturated mainly in workweek during morning and evening peak hours. The bottlenecks on these roads are caused by a small throughput of intersections and occasional accidents. The peripheral roads are almost fully equipped with detectors and each important intersection is controlled by traffic signal light. The data are measured at 90 second intervals and the traffic flow is characteristic by sudden changes of the measured intensities or occupancies.

The long-term overload of peripheral roads can cause damages of detectors. Although the micro-region is fully covered by detectors, it often happens that some of the detectors are broken. In this example, a real data coming from Prague city district Zličín, illustrated in Fig. 4, was used and the detector failure on one arm for 500 samples (about half a day) was simulated.

The proposed solution of such problem is based on the so called fictitious detector (FD), which can be understood as an expected course of the traffic flow on the particular arm. For each arm the FD is available but it is active only
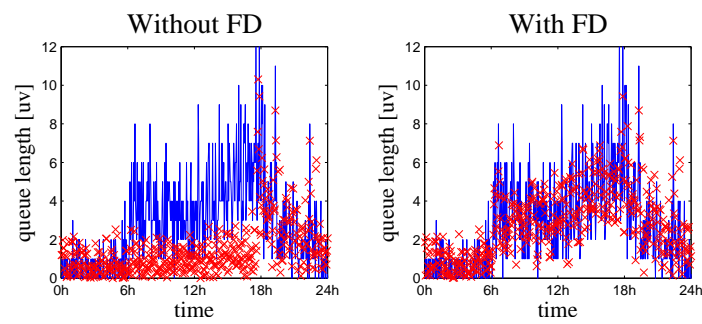

Fig. 5. The true (-) and estimated (x) queue length in case that detector failed with respect to application of the fictitious detector.

if the real detector got broken. The detector is supposed to be broken if the difference between measured intensity and expected intensity given by the FD is greater than a threshold and the output of the particular real detector is close to zero. In this case, the threshold was set for all detectors on value 5 vehicles per sample period. The impact of the FD on the resulting estimates is shown in Fig. 5, where the estimates are acquired by the DD1. The resulting estimates on the basis of the FD output are not so exact as the estimates on the basis of actually measured data but the FD significantly improve the estimates with respect to the estimates on the basis of data from failed detector.

\section{City Ring Road}

City ring roads are typically high-capacity roads where traffic intensity is high during a whole day. City ring roads are not usually equipped with traffic lights. The bottlenecks on this road are caused by reconstructions, accidents, and tunnels, which usually reduce the number of lanes. Currently, there is problem with outer Prague ring road which is still not completed and so the capacity is varying due to adaptations, which causes the congestion. With respect to the high traffic intensity, the queues can be long several hundreds of meters. Such queue influences the whole traffic network, for example, by blocking access roads. Monitoring of current queues and prediction of their future behaviour allows timely control action that influences the input intensity. The true queue lengths are unfortunately not available thus the queue lengths should be estimated.

City ring road and all important input or output roads are fully equipped with detectors. Data are measured at fiveminute intervals. The traffic flow is relatively constant without any sudden changes and thus, the KF can be used for queue lengths estimation. However, in various unexpected situations we need to adapt the model and so the DD1 is preferred. In this example it will be demonstrated that the estimates could be used for e.g., accident detection.

The estimation of queue length was tested on part of Prague city ring road Štěrboholská spojka with two-lane road in each direction, on which the main motorways of the Czech Republic are connected. The selected place on the city ring road is a black spot because of high traffic intensity and its closeness to interchanges. In this case, there are no further slip roads and no near parallel roads. Fig. 6 shows the chosen part with one lane obstructed by an accident in one direction on place where the ring road and slip roads are connected. 


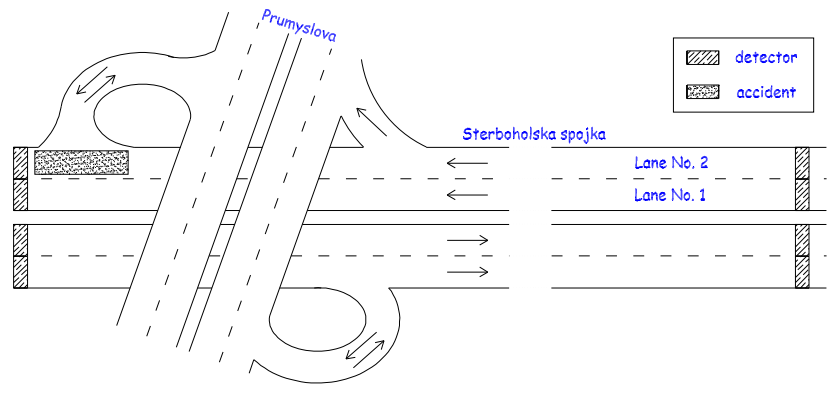

Fig. 6. Illustration of the chosen part of Prague city ring.
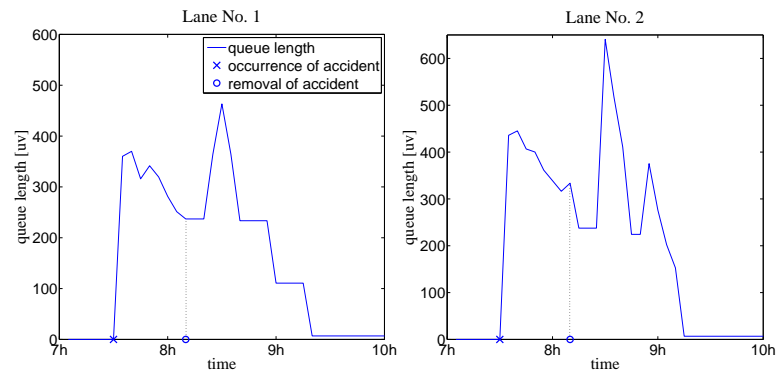

Fig. 7. The estimates of queue lengths caused by traffic accident on both lanes by means of the DD1.

Fig. 7 depicts the impact of an accident on the usually smooth traffic flow. The accident occurred during morning rush hours on lane No. 2, when the intensity of the traffic flow is almost equal to the capacity of the road. This accident caused that the number of arriving vehicles is greater than the capacity of the road with accident and thus the queue is formed. From the queue lengths estimates in the figure it can be seen that both queues are nearly the same (lane No. 2 is slightly more jammed), which corresponds with typical behaviour of drivers. The maximal queue length is formed a few minutes after removing the accident. This is due to the fact the speed of platoon of vehicles before the accident removal is significantly decreased and thus, the vacation of the road is not as fast as possible. In other words, the forty-minute accident, which blocks one lane, influences the traffic for two hours. This information is very important for traffic control in whole city because it influences not only vicinity of the city ring roads.

This example demonstrated the usefulness of the estimation to the unexpected situation detection. It was clearly shown that the estimates indeed reflect the occurrence of the accident by sudden raising of the queue lengths estimates.

\section{CONClusion}

The paper dealt with the application of state and parameter estimation techniques into the area of the traffic control problem. Technique for modelling traffic system was used for three different types of the traffic micro-regions, namely city ring road, peripheral roads, and city centre roads. For estimation of directly immeasurable queue lengths two approaches were used. First one was based on the off-line identification of unknown parameters and subsequent application of the Kalman filter and second one on simultaneous on-line estimation of the state and parameters leading to the nonlinear estimation techniques, namely to the derivative-free Kalman filters. It was shown that the Kalman filter is suitable for micro-regions with rather smooth traffic flow where all important roads are equipped with the detectors. For micro-regions with some immeasurable roads, with often accidents or with suddenly changing traffic flow, the simultaneous estimation of the state and parameters by the nonlinear filters is advantageous. In these cases, the divided difference filter first order seems to be a reasonable choice with respect to the estimation quality and computational demands.

\section{REFERENCES}

[1] J. Moor, S. Mattingly, C. MacCarley, and M. McNally, "Anaheim advanced traffic control system filed operation test: A technical evaluation of scoot," Transportation Planning and Technology, vol. 28, no. 6, pp. 465-482, 2005.

[2] E. Kosmatopoulos and et al., "International comparative field evaluation of a traffic-responsive signal control strategy in three cities," Transportation research part A - policy and practice, vol. 40, no. 5, pp. 399-413, 2006.

[3] B. Chilukuri, J. Perrin, and P. Martin, "SCOOT and incidents - performance evaluation in simulated environment," Transporattion research record, no. 1867, pp. 235-258, 2004.

[4] R. Pearson, "Traffic signal control," ITS Decision Report, 2000.

[5] T. Tichý, "Evaluation methodology for traffic control system," in Preprints of the ITS Prague '07 (in Czech), Prague, May 2007.

[6] J. Homolová and I. Nagy, "Traffic model of a microregion," in Preprints of the 16th IFAC World Congress, Prague, Czech Republic, July 2005, pp. 1-6.

[7] P. Pecherková, J. Homolová, and J. Duník, "Estimation of state and parameters of traffic system," in Proceedings of the Fourth International Conference on Informatics in Control, Automation and Robotics, Angers, France, May 2007, pp. 223-228.

[8] B. D. O. Anderson and S. B. Moore, Optimal filtering. New Jersey: Prentice Hall Ins.: Englewood Cliffs, 1979.

[9] S. J. Julier and J. K. Uhlmann, "Unscented filtering and nonlinear estimation," Proceedings of the IEEE, vol. 92, no. 3, pp. 401-421, 2004.

[10] M. Nørgaard, N. K. Poulsen, and O. Ravn, "New developments in state estimation for nonlinear systems," Automatica, vol. 36, no. 11, pp. 1627$1638,2000$.

[11] D. L. Alspach and H. W. Sorenson, "Nonlinear bayesian estimation using gaussian sum approach," IEEE Transactions on Automatic Control, vol. 17, no. 4, pp. 439-448, 1972.

[12] J. Duník, M. Simandl, O. Straka, and L. Král, "Performance analysis of derivative-free filters," in Proceedings of the 44th IEEE Conference on Decision and Control, and European Control Conference ECC'05, Seville, Spain, 11 2005, pp. 1941-1946.

[13] L. Ljung, System identification: theory for the user. UpperSaddle River, NJ: Prentice-Hall, 1999.

[14] E. A. Wan, R. van der Merwe, and A. T. Nelson, "Dual estimation and the unscented transformation," in Advances in neural Information Processing Systems 12. MIT Press, 11 2000, pp. 666-672.

[15] S. J. Julier, J. K. Uhlmann, and H. F. Durrant-White, "A new method for the nonlinear transformation of means and covariances in filters and estimators," IEEE Transactions On Automatic Control, vol. 45, no. 3, pp. 477-482, 2000.

[16] R. Mehra, "Approaches to adaptive filtering," IEEE Transactions on Automatic Control, vol. 17, no. 10, pp. 693-698, 1972.

[17] S. Verdú and H. Poor, "Minimax linear observers and regulators for stochastic systems with uncertain second-order statistics," IEEE Transactions On Automatic Control, vol. 29, no. 6, pp. 499-511, 1984.

[18] M. Šimandl and J. Duník, "Off-line estimation of system noise covariance matrices by a special choice of the filter gain," in Proceedings of the IEEE International Symposium on Intelligent Signal Processing, Alcalá de Henares, Spain, October 2007.

[19] Transport Simulation Systems, "Aimsun: Users manual," 2004, url: http://www.aimsun.com/. 